\section{Case}

Report

\title{
A Resectable Pancreatic Metastasis from Pulmonary Adenocarcinoma
}

\author{
Hitoshi Igai, MD, PhD, Mitsuhiro Kamiyoshihara, MD, PhD, Toshiteru Nagashima, MD,
} Yoichi Ohtaki, MD, PhD, and Kimihiro Shimizu, MD, PhD

\begin{abstract}
A 67-year-old man, diagnosed as primary pulmonary adenocarcinoma by intraoperative fine-needle aspiration biopsy cytology, underwent right lower lobectomy with radical lymphadenectomy. The pathological stage was Stage IIA (pT1bN1M0, N-reason: 12L positive). After surgery, nodular shadows without intrathoracic lymph node or distant metastasis were demonstrated metachronously three times by follow-up CT. Wedge resection was performed for each of the tumors, and the pathological diagnosis in each case was primary pulmonary adenocarcinoma, Stage IA (T1b), IA (T1a) and IA (T1a), respectively.

Five years after the initial pulmonary resection, a follow-up abdominal CT revealed a 20-mm nodular shadow. We suspected that this pancreatic tumor might be a primary rather than metastatic one, therefore, pancreatoduodenectomy was performed. Pathological examination revealed adenocarcinoma that was positive for thyroid transcription factor (TTF)-1, allowing a final diagnosis of metastatic pulmonary adenocarcinoma. This case is very rare, because most cases of pancreatic metastasis from lung cancer have already widespread disease at the time of diagnosis.

This case illustrates that pancreatic metastasis from pulmonary adenocarcinoma should be borne in mind, even if the pancreatic tumor is a solitary lesion without additional organ metastasis.
\end{abstract}

Keywords: pancreatic metastasis, pulmonary adenocarcinoma

\section{Introduction}

Although primary lung cancer frequently metastasizes to distant organs, the pancreas is a relatively infrequent site of metastasis in comparison with the brain, bone, and adrenal gland. Maeno and colleagues ${ }^{1)}$ reported that 26 of $850(3.1 \%)$ patients with primary lung cancer developed synchronous or metachronous pancreatic metastasis. In their study, while $10.5 \%$ of

Department of General Thoracic Surgery, Maebashi Red Cross Hospital, Gunma, Japan

Received: May 28, 2012; Accepted: June 25, 2012 Corresponding author: Hitoshi Igai, MD, PhD. Department of General Thoracic Surgery, Maebashi Red Cross Hospital, 3-2136 Asahi-cho, Maebashi, Gunma 371-0014, Japan

Email: hitoshi-iga@hotmail.co.jp

(C)2014 The Editorial Committee of Annals of Thoracic and Cardiovascular Surgery. All rights reserved. patients with small cell carcinomas had pancreatic metastasis, only $2.3 \%$ of patients with adenocarcinomas did so. Therefore, pancreatic metastasis from pulmonary adenocarcinoma is comparatively rare in comparison with small cell carcinoma. Additionally, as most patients with pancreatic metastasis have additional organ metastases, resectable cases are clinically rare. To our knowledge, only Mori's report ${ }^{2)}$ has demonstrated the case underwent radical surgical resection for both primary lung cancer and pancreatic metastasis.

Here, we report a resectable case of pancreatic metastasis from pulmonary adenocarcinoma with a successful result and review the relevant literature.

\section{Case Report}

A 67-year-old man was examined at our hospital for follow-up of an unrelated disease. Chest 


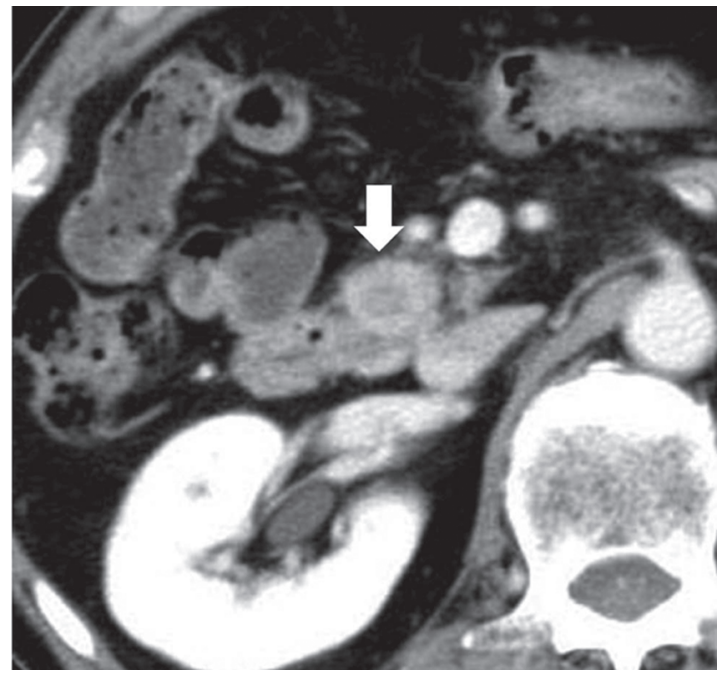

Fig. 1 Dynamic CT revealed a dense enhancement in only the peripheral area, in the early phase, suggesting a hypovascular tumor (arrow).

computed tomography (CT) demonstrated a 25-mm nodular shadow in the right lower lobe. Although transbronchial lung biopsy was performed, the diagnosis could not be confirmed. However, as we suspected that the nodular shadow was malignant disease, surgical resection including biopsy was performed to confirm the diagnosis. Intraoperative fine-needle aspiration biopsy cytology revealed that the nodule was adenocarcinoma, and, therefore, a right lower lobectomy with systemic lymphadenectomy was performed. The pathological stage was Stage IIA (pT1bN1M0, N-reason: $12 \mathrm{~L}$ positive). About 22, 42 or 53 months after 1 st lung resection, nodular shadows without intrathoracic lymph node or distant metastasis were demonstrated metachronously three times by follow-up CT. Wedge resection was performed for each of the tumors, as they were localized in the periphery of the ipsilateral lung. The pathological diagnosis in each case was primary pulmonary adenocarcinoma, because each tumor had peripheral bronchoalveolar pattern and central invasive adenocarcinoma pattern, respectively, Stage IA (T1b), IA (T1a) and IA (T1a), respectively.

Five years after the initial pulmonary resection, a follow-up abdominal CT revealed a $20-\mathrm{mm}$ nodular shadow, which was suspected to be a primary or metastatic pancreatic tumor. Dynamic CT revealed dense enhancement of only the peripheral area in the early phase, suggesting a hypovascular tumor (Fig. 1). Positron emission tomography with $\left[{ }^{18} \mathrm{~F}\right]$ fluoro-2-deoxy-D-glucose (FDG-PET)/CT revealed

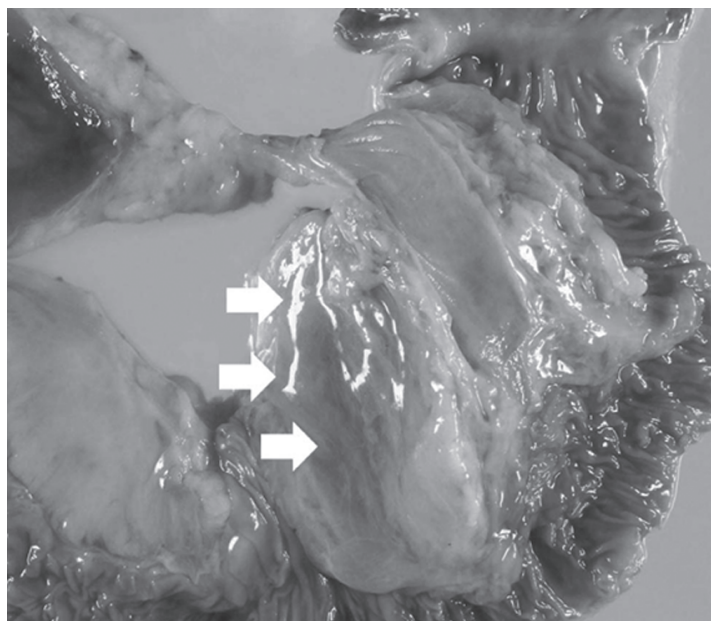

Fig. 2 The resected specimen contained a 16-mm tumor located in the head of the pancreas (arrows).

no abnormal FDG accumulation in other organs, and the SUVmax of the pancreatic tumor was 5.1.

In addition to the difficulties to confirm the diagnosis by endoscopic biopsy for this pancreatic tumor, we suspected that this pancreatic tumor might be a primary rather than metastatic one. Therefore, pancreatoduodenectomy was performed.

The resected specimen contained a 16-mm tumor located in the head of the pancreas (Fig. 2). Pathological examination revealed adenocarcinoma that was positive for thyroid transcription factor (TTF)-1, allowing a final diagnosis of metastatic pulmonary adenocarcinoma (Fig. 3). The postoperative course was uneventful, and the patient was discharged on postoperative day 30 . He remains disease free for an additional 6 months by radiographic findings.

\section{Discussion}

Pancreatic metastasis from any malignancy is clinically rare. Adsay and colleagues reported that 81 of 4955 autopsied patients (1.6\%) had metastatic pancreatic tumors. ${ }^{3)}$ Clinical studies have also revealed that pancreatic metastasis is rare among patients with solitary pancreatic masses and that the most common primary tumor responsible is renal cell carcinoma, followed by lung and colorectal cancer. ${ }^{4)}$

In the present case, preoperatively we suspected that the tumor was a primary pancreatic cancer for the following reasons:

1. Although the first pulmonary adenocarcinoma was p-Stage IIA, 5 years had passed. 


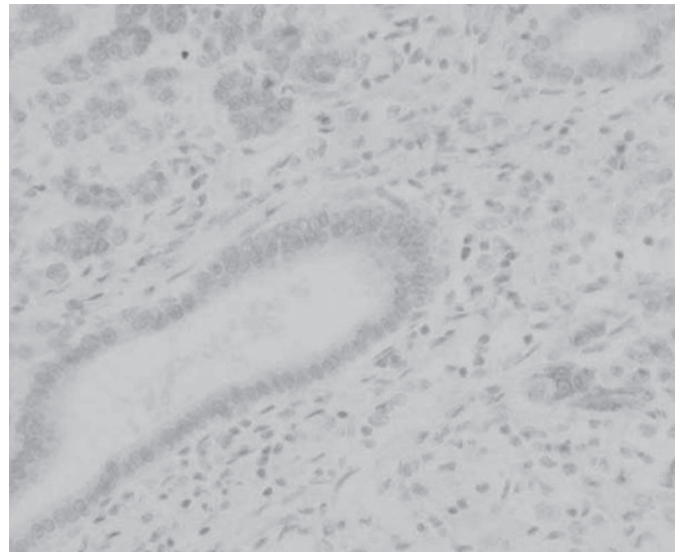

Fig. 3 Pathological examination revealed a TTF-1 positive adenocarcinoma, allowing a final diagnosis of metastatic pulmonary adenocarcinoma.

2. The 2nd, 3rd, and 4th adenocarcinomas were p-Stage IA, which is the earliest stage in the TNM classification.

3. The pancreas is a relatively uncommon site of metastasis from lung cancer, and isolated pancreatic metastasis from lung cancer without widespread disease is very rare.

However, the pathological diagnosis was metastatic pancreatic cancer from pulmonary adenocarcinoma. This illustrates the clinical difficulty in diagnosing primary or metastatic pancreatic tumors. Distinction between a metastatic and a primary tumor on the basis of only imaging modalities including CT, endoscopic retrograde cholangiopancreatography or ultrasonography is difficult, especially when a solitary mass is observed. ${ }^{5,6)}$

In patients with pancreatic metastases from renal cell carcinoma, a median survival period of 8.75 years was reported for 115 patients undergoing pancreatic resection. ${ }^{6)}$ In contrast, the usefulness of surgical resection for pancreatic metastasis from lung cancer is difficult to assess because of the rarity of this type of lesion. Additionally, most cases of pancreatic metastasis from lung cancer are unresectable at the time of diagnosis because the disease is already widespread. Therefore, the usefulness of surgical resection for pancreatic metastasis from lung cancer has remained unclear. However, several authors have reported successful resection of pancreatic metastasis from lung cancer. ${ }^{7,8)}$ Wilson et al. ${ }^{7)}$ reported a patient who remained diseasefree for 22 months after surgical resection for pancreatic metastasis from pulmonary adenocarcinoma, although the primary lung lesion was not resected because of disease progression. Mori, et al. ${ }^{2)}$ reported a patient had no disease recurrence for 24 months after surgical resection of pancreatic metastasis. As these were both simply case reports, they were unable to strongly support the validity of surgical resection for pancreatic metastasis from lung cancer, although they indicated the usefulness of surgical resection in appropriately selected patients. Except for Mori's report ${ }^{2)}$ and the present case, there have been no previous reports in English documenting radical surgical resection for both primary lung cancer and pancreatic metastasis. Therefore, the present rare case has to be followed-up in the prognosis.

In conclusion, isolated pancreatic metastasis from pulmonary adenocarcinoma without widespread disease should be borne in mind, even though its clinical diagnosis is difficult. Pancreatic resection for metastatic disease may be appropriate for selected patients, but its indications should be considered carefully.

\section{Disclosure Statement}

The authors have no conflict of interest to declare.

\section{References}

1) Maeno $T$, Satoh $H$, Ishikawa $H$, et al. Patterns of pancreatic metastasis from lung cancer. Anticancer Res 1998; 18: 2881-4.

2) Mori N, Sawada T, Satoh H, et al. A resected case of solitary pancreatic metastasis from adenocarcinoma of the lung. JOP 2008; 9: 698-703.

3) Adsay NV, Andea A, Basturk O, et al. Secondary tumors of the pancreas: an analysis of a surgical and autopsy database and review of the literature. Virchows Arch 2004; 444: 527-35.

4) Rumancik WM, Megibow AJ, Bosniak MA, et al. Metastatic disease to the pancreas: evaluation by computed tomography. J Comput Assist Tomogr 1984; 8: 829-34.

5) Swensen T, Osnes M, Serck-Hanssen A. Endoscopic retrograde cholangio-pancreatography in primary and secondary tumours of the pancreas. Br J Radiol 1980; 53: $760-4$

6) Wernecke K, Peters PE, Galanski M. Pancreatic metastases: US evaluation. Radiology 1986; 160: 399-402.

7) Reddy S, Wolfgang CL. The role of surgery in the management of isolated metastases to the pancreas. Lancet Oncol 2009; 10: 287-93.

8) Wilson RL, Brown RK, Reisman D. Surgical resection for metastatic non-small cell lung cancer to the pancreas. Lung Cancer 2009; 63: 433-5. 